\title{
Erratum: Self-Organization, Structures, and Anomalous Transport in Turbulent Partially Magnetized Plasmas with Crossed Electric and Magnetic Fields [Phys. Rev. Lett. 122, 185001 (2019)]
}

Oleksandr Koshkarov®, Andrei Smolyakov, Yevgeny Raitses, and Igor Kaganovich

Q (Received 8 November 2019; published 3 December 2019)

DOI: 10.1103/PhysRevLett.123.239903

We have found an unfortunate error in reporting actual parameters used in the simulations. The dimensionless parameters used for all calculations presented in this Letter are $u_{0}=241.8 c_{s}, v_{0}=3.72 c_{s}, v_{*}=-48.8 c_{s}, \nu=0.28 \omega_{\mathrm{LH}}$, $\sqrt{m_{i} / m_{e}}=427$, which correspond to the following dimensional parameters $E=10^{4} \mathrm{~V} / \mathrm{m}, B=100 \mathrm{G}, T_{e}=17.7 \mathrm{eV}$, $v_{0}=15 \mathrm{~km} / \mathrm{s}, u_{0}=10^{6} \mathrm{~m} / \mathrm{s}, \nu=1.15 \mathrm{MHz}, v_{*}=-2 \times 10^{5} \mathrm{~m} / \mathrm{s}, L_{n}=8.8 \mathrm{~mm}$.

Another related inconsistency is that there has to be a negative sign in front of the drift term $k_{y} v_{*}$ in Eq. (4). The drift velocity is negative $v_{*}=-\rho_{e}^{2} \omega_{c e} / L_{n}<0$, and Eq. (1) remains unchanged. Other results and figures remain unchanged. We thank S. Sadouni and G. Hagelaar for bringing our attention to this mistake. 